\title{
ARRHYTHMIAS
}

\section{Benefits of yoga in patients with atrial fibrillation}

The impact of yoga on atrial fibrillation (AF) burden has been assessed in a small, single-centre, proof-of-concept study of 49 patients with symptomatic paroxysmal AF. The Yoga My Heart study investigators believe that their findings "constitute the

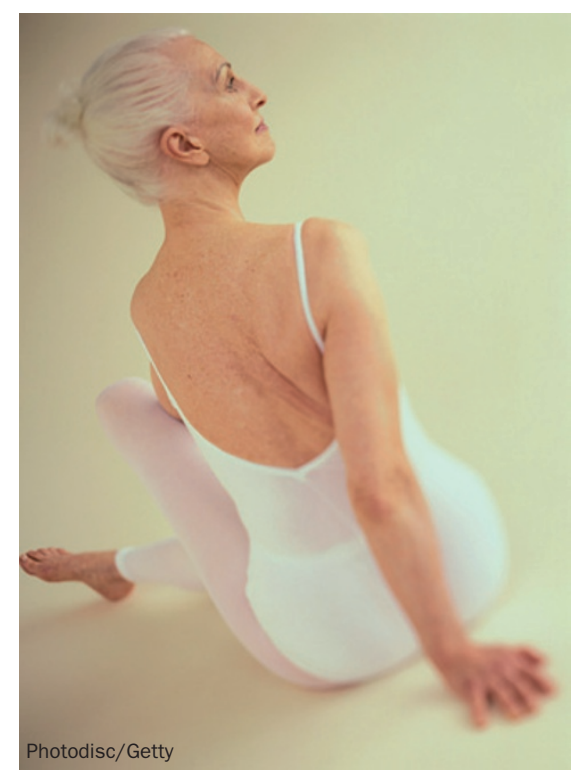

first evidence that yoga is effective as a complementary therapy for alleviation of AF burden and consequences".

The study involved a 3-month control period followed by a 3-month period, in which patients participated in an Iyengar yoga class at least twice weekly and were encouraged to practice postures at home on a daily basis. Three of the 52 enrolled patients withdrew from the study during the yoga intervention period. Of the patients who completed the trial, $47 \%$ were male. At baseline, mean age and BMI were 61 years and 28 , respectively, and mean duration of AF was 64 months.

Yoga was associated with reductions in the mean number of symptomatic AF episodes (2.1 vs 3.8 in the control period), symptomatic non-AF episodes (1.4 vs 2.9), and nonsymptomatic AF episodes (0.04 vs 0.12 ) over 3 months $(P<0.001$ for all). Almost one-quarter (11) of patients with AF during the control period experienced no AF episodes during the yoga training.

Yoga was also associated with improvements in quality of life, and reductions in anxiety and depression. Notably, the changes in anxiety and depression did not correlate with the changes in the symptomatic and nonsymptomatic AF episodes nor the symptomatic non-AF episodes.

In their study report, the investigators comment that "the benefit from the emotionally supportive atmosphere at yoga training centres, and the positive impact by the caring relationships, change in diet and lifestyle modification associated with yoga practices on physiological parameters cannot be underestimated". They conclude that "future large, focussed, randomized, controlled studies will be necessary to examine the ... mechanisms through which yoga may exert beneficial effects in patients with AF".

Bryony M. Mearns

Original article Lakkireddy, D. et al. Effect of yoga on arrhythmia burden, anxiety, depression, and quality of life in paroxysmal atrial fibrillation: the YOGA My Heart study. J. Am. Coll. Cardiol. doi:10.1016/j.jacc.2012.11.060 